\title{
An experimental investigation of microresistor laser printing with gold nanoparticle-laden inks
}

\section{Journal Article}

\section{Author(s):}

Bieri, N.R.; Chung, J.; Poulikakos, D.; Grigoropoulos, C.P.

Publication date:

2005-04

Permanent link:

https://doi.org/10.3929/ethz-b-000033461

Rights / license:

In Copyright - Non-Commercial Use Permitted

Originally published in:

Applied Physics A 80(7), https://doi.org/10.1007/s00339-004-3195-8 


N.R. BIERI
J. CHUNG $^{2,3}$
D. POULIKAKOS
C.P. GRIGOROPOULOS
3

\section{An experimental investigation of microresistor laser printing with gold nanoparticle-laden inks}

\footnotetext{
${ }^{1}$ Laboratory of Thermodynamics in Emerging Technologies, Institute of Energy Technology, ETH Swiss Federal Institute of Technology, ETH Zentrum, 8092 Zurich, Switzerland

${ }^{2}$ Department of Mechanical Engineering, Korea University, Seoul, Korea

${ }^{3}$ Department of Mechanical Engineering, University of California, Berkeley, CA 94720, USA
}

\begin{abstract}
Received: 15 November 2004/Accepted: 14 December 2004 Published online: 17 February 2005 • (C) Springer-Verlag 2005

ABSTRACT This paper presents an experimental investigation of the novel thermal manufacturing process of printing and laser curing of nanoparticle-laden inks that can produce functional microstructures such as electronic microresistors and interconnections for semiconductors and other devices. Of specific interest are the complex and interweaved transport phenomena involved, focusing on the absorption and diffusion processes of irradiated laser energy influencing solvent vaporization, the nanoparticle curing process, the substrate, and the final quality of the produced resistor. Parametric studies of the thermal process together with extensive microscopy analysis of the topography and resistivity measurements piece together a better understanding of the underlying physics and aid the development of the technology.
\end{abstract}

PACS 81.16.Mk; 81.16.Rf

\section{Introduction}

Driven by the continuous need for advancement in state of the art micro- and nanomanufacturing in a host of fields, exemplified prominently by electronics, new methodologies and processes need to be developed, after overcoming formidable scientific challenges, to engineer nanostructures with desired properties and functionality. At the same time, the emergence of consistent manufacturing methods of ultra fine particles (UFP) (or, after the more popular terminology nanoparticles) of many materials, is creating detailed activity related to their utilization. The reason for using nanoparticles in this work lies in the significant melting temperature depression compared to their bulk counterpart [1]. This work introduces and investigates a novel technique for producing electrically conductive gold lines by low temperature laser induced curing of nanoparticle suspensions. Drop-on-demand (DOD) ink jet printing is utilized to print the nanoparticles, which are suspended in a carrier-liquid of so called nanoink, into desired micro-structures. A laser with an appropriately selected wavelength [2] is used to melt the nanoparticles to produce a continuous, electrically conducting line. Instead of heating/baking the whole substrate in the oven, a laser is

Fax: +41-1-632-11-76, E-mail: dimos.poulikakos@ethz.ch used to melt and sinter the nanoparticles to form a continuous line and therefore only elevates the temperature locally and in a controlled manner, thereby protecting thermally sensitive electrical components on the substrate and limiting the built up of thermal stresses. The feature size is not defined by the size of the printed line but by the focal diameter of the laser and can be decreased down to micrometers in size by a tight focusing of the laser.

The work presented herein involves the drop-on-demand printing of suspensions (inks) of gold nanoparticles and the manufacturing of gold conductors by laser curing with an appropriately selected wavelength. The gold structures produced are analyzed by different optical and mechanical methods and the achieved resistivities are compared to those of bulk gold. Studies are also conducted to determine useful parametric domains.

\section{Experimental}

\section{Microdroplet generation and visualization}

The microdroplet generation and deposition of the nanoink, consisting of $30 \mathrm{wt} . \%$ gold, $50 \sim 70 \mathrm{wt} . \%$ toluene, under $10 \mathrm{wt} \%$ copper compound (as $\mathrm{CuO} / \mathrm{Au}$ ), under 10 wt. $\%$ bismuth compound (as $\mathrm{Bi}_{2} \mathrm{O}_{3} / \mathrm{Au}$ ), and a trace of interfacial active agent to prevent agglomeration of nanoparticles (Vacuum Metallurgical, Au102T), is accomplished with an appropriately modified piezoelectrically driven microdroplet jetting device consisting of a high temperature piezoelectric jetting device (Microfab) with an orifice diameter of $60 \mu \mathrm{m}$. This piezoelectric jetting device allows for the generation of monodispersed droplets on demand with diameters in the range of $60-100 \mu \mathrm{m}$. The procedure of generating a droplet can be described as follows. By applying a bipolar electric pulse (Fig. 1) to a piezoelectric ceramic tube enclosing a glass capillary containing the nanoink, a microdroplet is ejected on demand. The glass capillary has an inner diameter of approximately $500 \mu \mathrm{m}$ and an orifice diameter of $60 \mu \mathrm{m}$ and is filled with the nanoink which is in contact with the fluid reservoir. The bipolar electric pulse shape (Fig. 1) is of particular importance for controlling the size, velocity, and stability of the generated droplets and especially for avoiding satellite droplets [3-7]. The parameters used are summarized in Table 1. The experimental setup for the microdroplet generation and deposition consisting of the piezoelectrically driven 


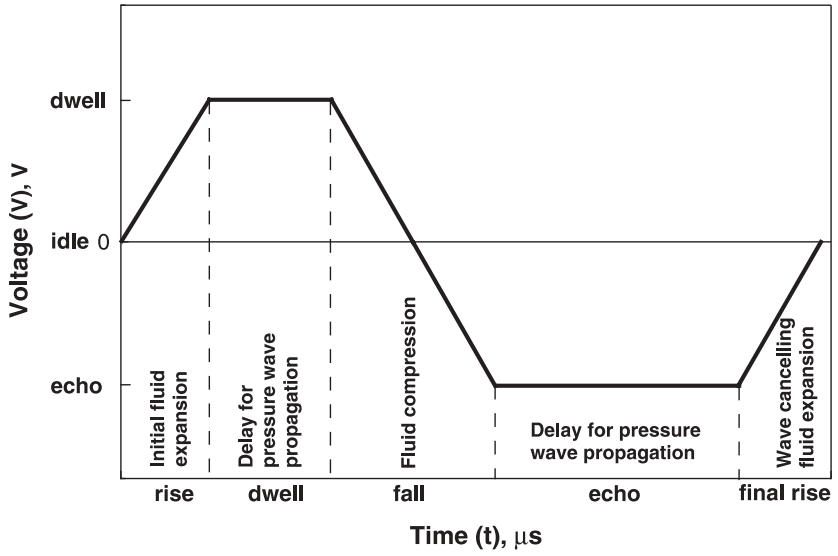

FIGURE 1 Bipolar electric pulse shape to drive the piezoelectric jetting device. The parameters used in the experiment are listed in Table 1

\begin{tabular}{lc}
\hline Parameter & Value \\
\hline Voltage idle $(\mathrm{V})$ & 0 \\
Voltage dwell $(\mathrm{V})$ & 36 \\
Voltage echo $(\mathrm{V})$ & -36 \\
Time interval rise $(\mu \mathrm{s})$ & 95 \\
Time interval dwell $(\mu \mathrm{s})$ & 112 \\
Time interval fall $(\mu \mathrm{s})$ & 95 \\
Time interval echo $(\mu \mathrm{s})$ & 220 \\
Time interval final rise $(\mu \mathrm{s})$ & 92 \\
Orifice diameter $[\mu \mathrm{m}]$ & 60 \\
Droplet diameter $[\mu \mathrm{m}]$ & $85-90$ \\
Droplet velocity $[\mathrm{m} / \mathrm{s}]$ & 1.8 \\
Printing frequency $[1 / \mathrm{s}]$ & 15 \\
\hline
\end{tabular}

TABLE 1 Parameters for the bipolar electric pulse shape (Fig. 1) to drive the piezoelectric jetting device and some printing parameter

DOD jetting device, a backpressure control, a de-clogging nitrogen backpressure system, and the equipment for the visualization is depicted in Fig. 2. The reservoir with the nanoink is sealed leak-proof and a low vacuum of the order of $10 \mathrm{mbar}$ is maintained in order to prevent the nanoink from leaking

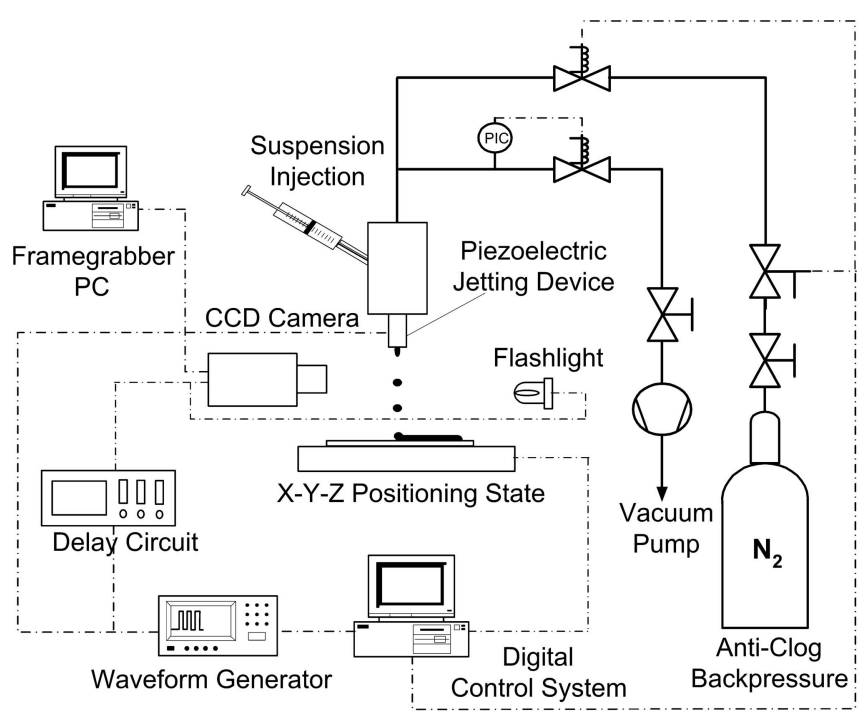

FIGURE 2 Experimental setup for the nanoparticle suspension droplet generation and deposition. Consisting of a piezoelectrically driven DOD jetting device, a backpressure control (vacuum pump), and a de-clogging nitrogen backpressure system and the equipment for the visualization out of the glass capillary tube due to the strong wettability of the glass capillary by toluene, the carrier-liquid of the gold nanoparticles. Because a constant extraction by suction would end up in successive evaporation of the toluene and a coagulation of the suspension, a magnetic valve (Vacuubrand $\mathrm{GmbH}, \mathrm{VV6C}$ ) with a vacuum control system (Vacuubrand, CVC2II) is installed between the reservoir and the vacuum pump. The capacity of the pump is regulated by two needle valves. During standstill periods the nanoink tends to clog the orifice. With a short nitrogen gas (Pangas) puff the orifice is cleared again. This cleaning procedure is carried out by a pressure regulating valve (Tescom, ER3000) to control the needed nitrogen backpressure (0.3-0.6 psi) and a magnetic valve (Lucifer) to produce a short impulse. The substrate is placed on an $x$-, $y$-, fast precision positioning stage ( $x$-direction: ALS 130-150, $y$-direction: ATS 100, AEROTECH) to print lines and various two-dimensional patterns. The distance, $z$, between the substrate and the orifice can be adjusted with a $z$-translation table (Linos). The uniform substrates (they are objective slides $26 \times 76 \times 1 \mathrm{~mm}$, Menzel) are fixed on a surface mounting placed on the $z$-translation stage. The jetting device and the positioning stage are controlled by a digital control system. Flash video microscopy $[3,5,8]$ is used to record and monitor the droplet generation and deposition process. In flash video microscopy only one picture is taken at one instant during a steady state droplet generation for each drop studied. Increasing the delay between the droplet ejection and the acquisition of each picture allows reconstruction of the entire process. The time resolution of the strobe technique is in the order of $0(1 \mu \mathrm{s})$. Additionally, the droplet velocity and size can be determined by the pictures. A triggered progressive scan CCD camera (JAI, M10) and a microscope objective (Microtech, Zoom 70) are used for the imaging. The backlighting is provided by a triggered xenon flash light (L4634, Hamamatsu) with a flash energy of $0.15 \mathrm{~J} /$ flash having a burst duration of $1 \mu \mathrm{s}$ with less than $200 \mathrm{~ns}$ jittering. The pictures are recorded with a framegrabber (Stemmer Imaging, ICIPCI). The droplet generation of a single droplet is initialized by a single electric pulse (drop-on-demand), having the bipolar shape of Fig. 1 to actuate the piezoelectric tube in the appropriate way. The pulse is generated by a waveform generator (LeCroy, LW420). Another pulse is generated by the waveform generator with a delay to trigger the strobe and the camera $[5,8,9]$.

\subsection{Experimental setup and procedure}

Figure 3 shows the experimental setup for the laser curing experiments consisting of two main parts, the DOD jetting device and the curing system. For simplicity, only the jet head and positioning stage are shown from the droplet generation setup. The laser curing assembly consists of a continuous single mode argon ion laser $\mathrm{Ar}^{+}$(Lexel, Model 3500-7, wavelength: $488-514 \mathrm{~nm}$ ). The laser beam is directed in two different ways to the experiment. In "configuration A" (Fig. 3a) a fiber coupler (Polytec, LVO 800) is used to couple the laser beam into the core of a fiber (Polytec, LVO-810/811) having collimating lenses at both ends for guiding the laser beam safely and easily to the substrate. The beam, with an initial diameter of $1.45 \mathrm{~mm}$ (defined where the intensity drops 


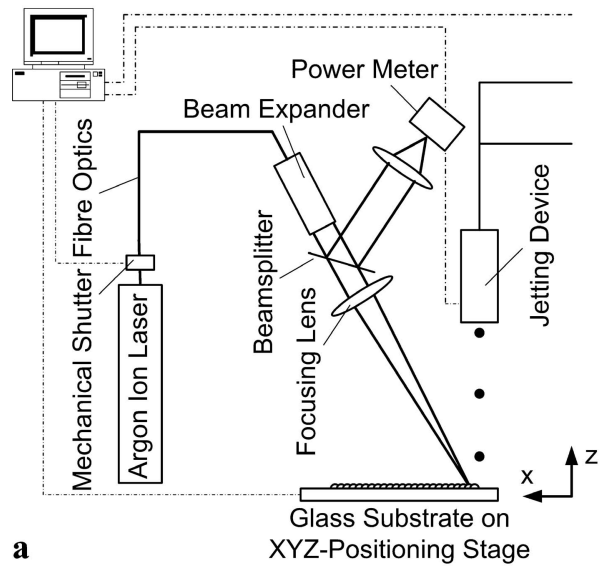

Control System

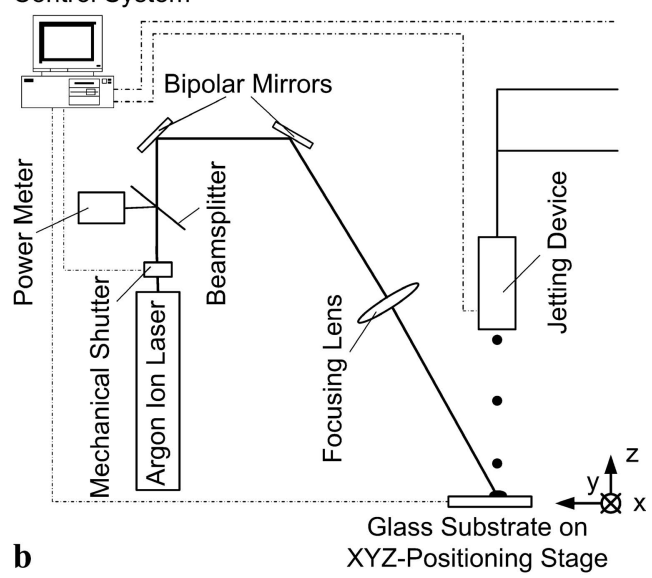

FIGURE 3 (a) Laser curing "configuration A": The laser beam is guided by an optical fiber to the substrate. The laser beam is in the $x-z$ plane. (b) Laser curing "configuration B": The laser beam is guided by mirrors to the substrate. The laser beam is in the $y-z$ plane. The translation direction is the $x$ direction by $1 / \mathrm{e}^{2}=0.135$, measured with knife edge) is expanded with a beam expander (Leica, beam expander with magnification $7 \mathrm{x}$ ) before focusing in order to achieve spot sizes in the range of 10 to $100 \mu \mathrm{m}$ with a long focal waist. The focal waist defines the length where the laser spot is constant (minimal) before expanding and is important for the positioning of the substrate. The substrate has to be planar in order to be at a constant distance from the lens otherwise the laser spot does not have the same size everywhere. The planar position is measured with an indicator caliper having an accuracy of $5 \mu \mathrm{m}$. A beam splitter is used to monitor the laser power (Coherent, FieldMaster GS) simultaneously.

The printing (including the positioning) and curing processes are synchronized by a digital control system. The control system controls the printing process and a mechanical shutter blocking the laser beam when closed, is placed between the argon laser and the fiber optic coupling system. The laser beam is focused on the printed line with an incident angle of $30^{\circ}$ parallel to the translation direction ( $x$-direction), preferably close to the point of droplet impact. In Fig. 3b "configuration B" is shown. The alternative setup is chosen because microstructures with a larger cross-sectional area are easier to investigate. The employment of a larger laser focal area requires more laser power which is not achievable with "configuration A". The main changes are therefore the replacement of the fiber optics (50\% power loss during coupling process) by dielectric mirrors (reflectance $>99 \%$ ) and the incidence direction of the laser which is perpendicular to the translation direction $x$. Analogously to "configuration A" a beam splitter is utilized to monitor the laser power and the printing and curing processes are synchronized by the same digital control system.

\section{3}

3.1

\section{Results}

\section{Analysis of the gold microstructures}

The main parameters influencing the manufacturing of the conducting microstructure are the intensity of the curing laser and the translation velocity (also called curing velocity herein) of the substrate. Gold conductors are cured with a laser power of $50-500 \mathrm{~mW}$ at two different velocities. The cured lines are analyzed by different methods such as scanning force microscope (SFM), scanning electron microscope (SEM), near field scanning optical microscope (SNOM), and energy dispersive X-ray analysis (EDX).

In the conducted experiments the beam diameter is always smaller than the width of the printed nanosuspension line which is defined by the reliably achievable droplet size and translation velocity of the substrate. The nanoparticle suspension line is displaced outward due to surface tension gradient induced by the heating of the laser focused in the middle of the printed nanoparticle suspension line. In addition, the liquid is displaced outward due to the evaporation of the carrier liquid during the curing process. The portion of uncured solution at the sides of the cured region can be washed away in an additional step by simply dipping the sample in a toluene bath.

The laser beam radius is $8.5 \mu \mathrm{m}$, (defined where the intensity drops by $1 / \mathrm{e}^{2}=0.135$, measured with knife edge), with wavelength $488 \mathrm{~nm}$ and the laser incident angle is $30^{\circ}$. The incidence plane of the laser is defined by the translation direction and the normal to the substrate (setup A). The printing and curing are performed simultaneously.

A scanning force microscope (SFM), a Topometrix Explorer, in contact mode, with a lateral resolution of micrometers and a vertical resolution of the order of Angstroms is used to obtain information about the topography, height, and cross sectional area needed, among other things, for the calculation of the resistivity of the produced gold conductor. In contact mode, the SFM measures the topography by rastering the probe tip over the sample while in contact with the sample surface. Figure 4 shows 3 -dimensional visualizations and cross-sectional profiles of the gold lines, cured with a laser power of $50 \mathrm{~mW}$ (Fig. 4a) and $300 \mathrm{~mW}$ (Fig. 4b) and a curing velocity of $1 \mathrm{~mm} / \mathrm{s}$. The scanned area is a square of $100 \mu \mathrm{m} \times 100 \mu \mathrm{m}$. The height of the structures is amplified in order to highlight the surface structure because the height of the surface structure is of the order of $10-1000 \mathrm{~nm}$. The topography of the $50 \mathrm{~mW}$ line (Fig. 4a) is very smooth except for the edges (note that the vertical scale in this figure is in nanometers). The edges feature, a structure of needlelike nature [10] is also present in the middle of the cured line for the case of higher power (Fig. 4b), but the physical mechanism is different. Representative cross-sectional areas of the gold conductors are shown in Fig. 4c and d. Both lines exhibit elevated rims at the edges and the evolution of the structure bears some similarity to the one described and inves- 

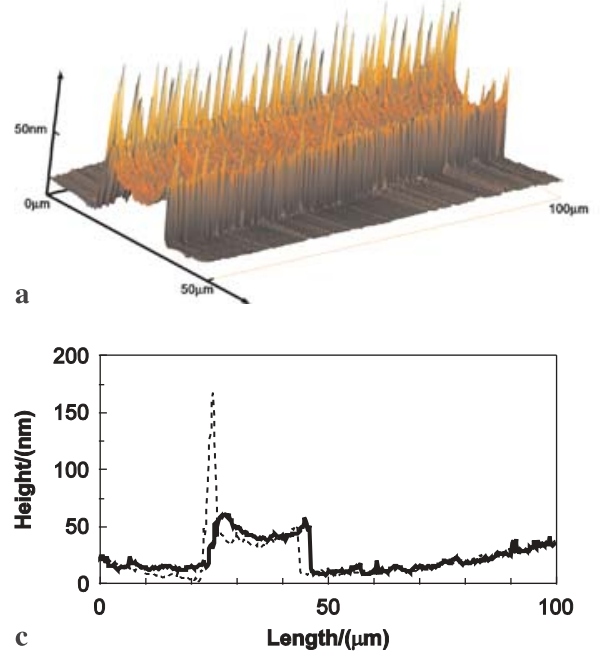
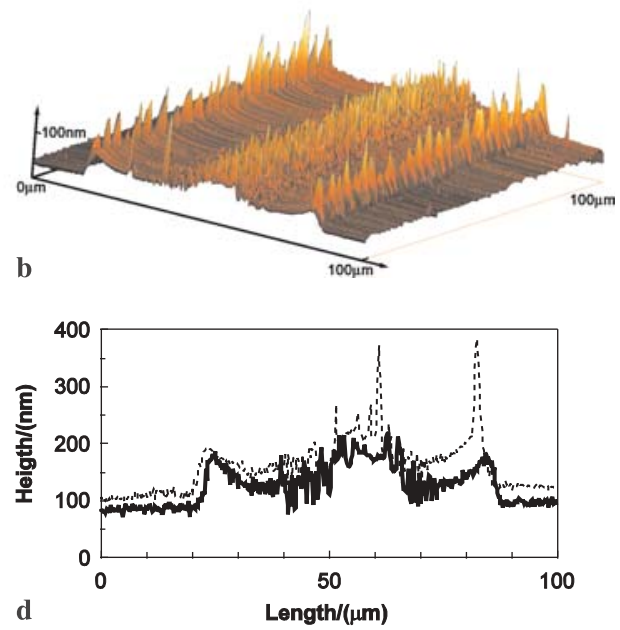

FIGURE 4 3-dimensional (SFM) visualization of gold lines cured with a laser beam radius of $8.5 \mu \mathrm{m}$, a curing velocity of $1 \mathrm{~mm} / \mathrm{s}$ and a laser power of (a) $50 \mathrm{~mW}$ and (b) $300 \mathrm{~mW}$. Both lines show elevated rims on both sides with needles on top. The scale of the pictures is not the same. More detailed information is given by the cross-section of the gold line cured with a laser power of (c) $50 \mathrm{~mW}$ and (d) $300 \mathrm{~mW}$. Typical cross-section (solid line), crosssection with nanoneedle peak (dashed line) tigated by Bennett et al. [11] regarding laser texturing of glass surfaces.

The profile in Fig. 4c is from now on called "bowl" shape and the profile in Fig. 4d "sombrero" shape. In-situ images of the curing process $[10,12,13]$ helped to explain the lateral rims and the curing mechanism in the following way, which is schematically outlined in Fig. 5: A highly reflective gold layer begins to form near the evaporation interface contact line ahead of the laser spot. The toluene evaporates due to laser radiation absorption in the gold layer and the subsequent thermal diffusion through the substrate and gold layer toward the evaporation interface. Due to the reduction of the surface tension (thermocapillarity) caused by the heating, a Marangoni flow evolves which displaces the ink ahead and around the scanning laser spot forming a U-shaped convex ink meniscus. The Marangoni flow also generates a circulation inside the nanoink, leading to, in conjunction with the evaporation, an increase in particle concentration in the vicinity of the contact line. This pool of particles supplies material to the edges of the

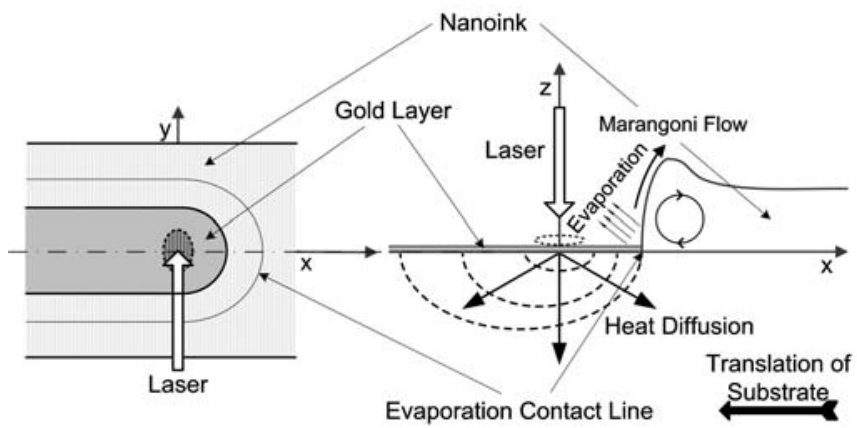

FIGURE 5 Schematic of the physics: A highly reflective gold layer begins to form near the evaporation contact line ahead of the laser spot. The toluene evaporates due to laser radiation absorption in the gold layer and the subsequent thermal diffusion trough the substrate and gold layer toward the evaporation interface. Due to the reduction of the surface tension (thermocapillarity) caused by the heating, a Marangoni flow evolves which displaces the ink ahead and around the scanning laser spot forming a U-shaped convex ink meniscus. The Marangoni flow also generates a circulation inside the ink and the concentration of nanoparticles is increased near the contact line. Gold nanoparticles are mainly deposited right at the evaporation interface contact line building the high rims. developing gold line, fostering rim formation and a distinct change in the line topography. The submicron scale needle structures with heights between $500 \mathrm{~nm}$ and $1000 \mathrm{~nm}$ periodically spaced at the rim, are also only present if the ink is displaced around the laser spot. Note that the length (microns) and height $(\mathrm{nm})$ scales in Fig. 4 are different. Although these needle structures are practically negligible compared to the width of the cured stripe, their presence is of scientific interest. A possible explanation of the formation of the needle microstructures is that convection micro-cells which are formed in the displaced ink delivering particles that are cured at the locations of the curing line where the needle microstructures appear. The upward motion of the toluene vapor should also influence the formation of these structures [10].

The two gold conductors are analyzed with scanning electron microscopy (SEM) and scanning near field optical microscopy (SNOM). For brevity the results are not shown here in detail $[2,12]$. The SEM showed a break-up of the continuous gold layer into fractal like structures in the disturbed middle region and into isolated agglomerated gold particles at increasing power, indicating that the bump in the center of the gold line (Fig. 4d) is not gold [2]. The SNOM pictures also confirmed the absence of a continuous gold layer in the bumpy center region [2]. The gold conductor is further investigated with energy-dispersive X-ray analysis (EDX) at two different locations, a) in the continuous gold layer, and b) in the bumpy center region on the material surrounding the isolated agglomerated gold particles. The composition of the different layers is shown in Table 2. The gold layer contains $\mathrm{Au}$ but also $\mathrm{Bi}$ and $\mathrm{Cu}$. Both $\mathrm{Bi}$ and $\mathrm{Cu}$ are components of the nanoparticle suspension and declared from the manufacturer (Vacuum Metallurgical). Because the electron beam has a certain penetration depth it is also possible to coanalyze the host material. This explains the presence of $\mathrm{Si}$, $\mathrm{Al}$, and $\mathrm{Fe}$ in the analysis of the gold layer because all the elements are part of the soda-lime glass (approx. composition: $72-74$ wt. $\% \mathrm{SiO}_{2}, \sim 15$ wt. $\% \mathrm{Na}_{2} \mathrm{O}, \sim 9$ wt. $\% \mathrm{CaO}$, $\sim 1-1.9$ wt. $\% \mathrm{Al}_{2} \mathrm{O}_{3}, \sim 0.09-0.1$ wt. $\% \mathrm{Fe}_{2} \mathrm{O}_{3}$ ). In the center region on the material surrounding the isolated agglomerated gold particles, no trace of gold nor carbon is present which could have been the material of the bump as a prod- 


\begin{tabular}{lcc}
\hline Element & Gold layer [wt. \%] & Centre region [wt. \%] \\
\hline $\mathrm{Al}$ & 0.5 & 0 \\
$\mathrm{Si}$ & 31.48 & 94.05 \\
$\mathrm{Cu}$ & 0.81 & 1.59 \\
$\mathrm{Mg}$ & 1.14 & 3.08 \\
$\mathrm{Fe}$ & 0.41 & 0.15 \\
$\mathrm{Au}$ & 61.58 & 0 \\
$\mathrm{Bi}$ & 4.08 & 1.14 \\
\hline
\end{tabular}

TABLE 2 EDX analysis of the two different regions (in wt. \% of the pure elements) (a) gold layer and (b) disturbed region in center region

uct of combustion of the toluene. The center region consists of 94 wt. \% Si. The $\mathrm{Cu}$ and $\mathrm{Bi}$ traces must be leftovers of the nanoparticle solution. In both cases, the presence of $\mathrm{Mg}$ could be explained as part of the soda lime glass, as some manufacturers add small amounts of $\mathrm{MgO}$. Nevertheless the detailed chemical composition of the objective slide from Mentzel (glass manufactured by Schott) is not given by the manufacturer.

The bump in the center of the gold line cured at high power consists of glass and, as will be definitively shown later in this paper, is caused by an expansion of the glass substrate that does not completely recover. This information is of high importance for the determination of the "real" cross sectional area which is used for the calculation of the resistivity, as including the area occupied by glass would lead to a serious over prediction of the latter.

The laser curing setup is changed to "configuration B" (Fig. 3b) and the laser beam radius is increased to $39 \mu \mathrm{m}$ and the wavelength to $514 \mathrm{~nm}$ for the following experiments to facilitate the analysis of the produced gold conductors. The coupled printing/curing process is decomposed into two independent processes which are carried out sequentially. In the coupled scheme (when the curing occurs on-line with the printing), the line printing and curing speeds are identical. By changing the curing velocity the printing velocity is changed, therefore the droplet generation frequency has to be adjusted to achieve the same droplet density impinging on the substrate. In the decoupled process the nanoink line is produced in a first step at a constant printing velocity with a constant droplet generation frequency of $30 \mathrm{~Hz}$, because it yields a stable stream of droplets. In a second step the nanoink line is cured with a different curing velocity. The printing velocity defines the resulting width and thickness of the nanoink line (Fig. 6). The substrates are, unless differently indicated, objective slides made of soda-lime glass (Menzel).

\section{2 \\ Parametric study: Resistivity as a function of process parameters}

The quality of the produced gold conductor is determined quantitatively by measuring the electrical resistance. The direct resistance measurement is a simple method, where an electrical potential is applied between the ends of the gold conductor and the current flow, $i_{\mathrm{m}}$, in the leading wire as well the potential, $U_{\mathrm{m}}$, over the conductor is measured simultaneously. The resistivity is defined as $\varrho=R_{\mathrm{T}} A / l(\Omega \mathrm{m})$, where $A$ and $l$ are the cross sectional area $\left(\mathrm{m}^{2}\right)$ and length of the conductor (m), respectively. The power supply (Yokogawa 2554) gives the electrical potential. A power meter (Schlumberger
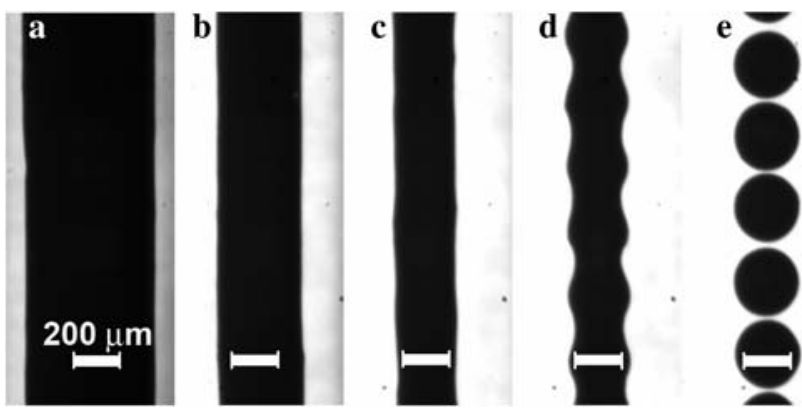

FIGURE 6 Evolution of the printed nanoink line from a wide continuous line composed from multiple overlapping droplets to single droplet with increasing printing velocity: (a) $1 \mathrm{~mm} / \mathrm{s}$, (b) $3 \mathrm{~mm} / \mathrm{s}$, (c) $6 \mathrm{~mm} / \mathrm{s}$, (d) $8 \mathrm{~mm} / \mathrm{s}$, and (e) $9 \mathrm{~mm} / \mathrm{s}$. The printing frequency is $30 \mathrm{~Hz}$, and the droplet size $85 \mu \mathrm{m}$

Solartron 7045) and a voltmeter (ESCORT EDM-2347) measure the current flow, $i_{\mathrm{m}}(\mathrm{A})$, and the potential, $U_{\mathrm{m}}(\Omega)$, respectively. By measuring the current without connecting the gold conductor, the fault current, $i_{\mathrm{d}}(\mathrm{A})$, is determined. The current running through the gold conductor, $i_{\mathrm{T}}(\mathrm{A})$, is the difference between the measured and the fault current. The resistance of the gold line is calculated using the following equations:

$$
\begin{aligned}
& R_{\mathrm{T}}=\frac{U_{\mathrm{m}}}{i_{\mathrm{T}}} \\
& i_{\mathrm{T}}=i_{\mathrm{m}}-i_{\mathrm{d}} .
\end{aligned}
$$

Firstly, the influence of the thickness of the pre-printed nanoink line on the final gold conductor is investigated by comparing the resulting cross-sectional area and resistivity of resulting gold lines. The nanoparticle suspension lines created with three printing velocities, 1,3 , and $6 \mathrm{~mm} / \mathrm{s}$ (Fig. 6), are cured with two laser powers, 0.6 and $1.2 \mathrm{~W}$ and different curing velocities. Figure 7 shows the cross-sectional areas and the resistivities of the gold lines as a function of the curing velocities for the three printing velocities. The profiles of the cross-sections are measured with a profilometer (Tencor P-10). At a low laser power (Fig. 7a), the cross sectional area decreases with increasing curing velocity. Since the temperature is lower, with a high curing velocity, the width of the semicircular contact line is getting smaller, which results in smaller deposition of nanoparticles. In addition, the porosity is larger due to less heating. Accordingly, the resistivity increases with the curing velocity (Fig. 7c). In Fig. 7d, at high laser power and a very low curing velocity, the resistivity is very high because isolated agglomerated gold particles are formed at the middle due to overheating. For the different printing velocities, the cross sectional areas do not change very much with a maximal error of $5 \%$ for the lines produced with very slow curing velocities of 0.05 and $0.1 \mathrm{~mm} / \mathrm{s}$ and $0.1 \%-3 \%$ for the others. At these slow curing velocities the substrate suffers high thermal damage resulting in a very rough surface, and hence in markedly different cross-sectional areas depending on the measuring spot. The cross-sectional areas of the lines cured with $0.6 \mathrm{~W}$ and with a printing velocity of $6 \mathrm{~mm} / \mathrm{s}$ seem to be slightly larger than the ones with printing velocities of 1 and $3 \mathrm{~mm} / \mathrm{s}$ (Fig. 7a). Nevertheless the deviation is less then $5 \%$ and the calculated resistivities do not show any difference compared to other printing velocities (Fig. 7c). 

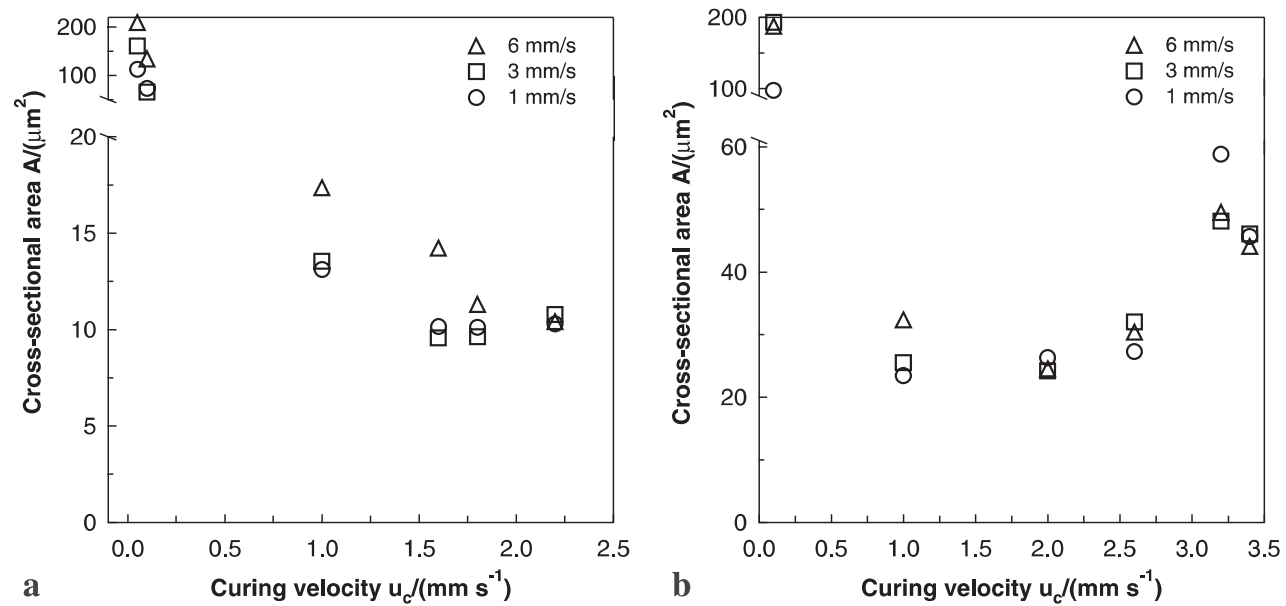

FIGURE 7 Cross-sectional areas and resistivities of gold lines cured with a laser beam radius of $39 \mu \mathrm{m}$, a laser power of (a), (c) $0.6 \mathrm{~W}$ and (b), (d) $1.2 \mathrm{~W}$ as a function of curing velocity for different printing velocities: $6 \mathrm{~mm} / \mathrm{s}$ (triangle), $3 \mathrm{~mm} / \mathrm{s}$ (square) and $1 \mathrm{~mm} / \mathrm{s}$ (circle)
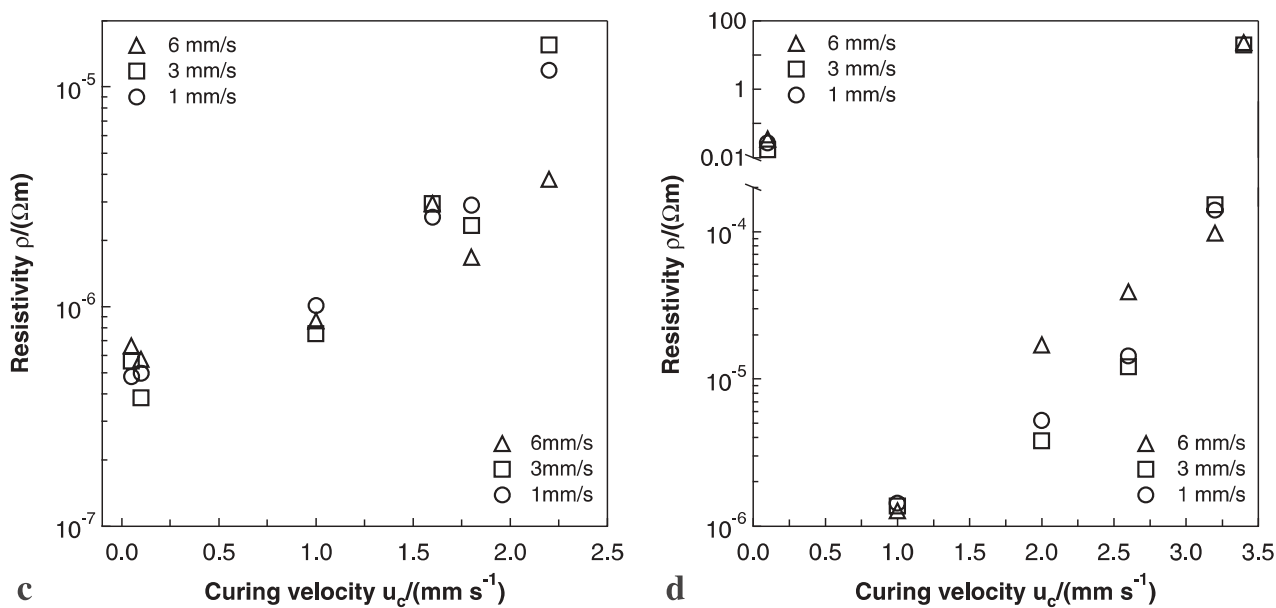

The calculation of the resistivity requires knowledge of the cross-sectional area $A$. A deformation of the substrate gives erroneous results of the effective resistivity. The deformation of the glass is therefore determined by profiling the gold conductor with and without the gold layer. After profiling the gold conductor, the gold layer is etched away in a bath of Königwasser, a solution composed of one part nitric acid and three parts hydrochloric acid, which does not affect glass. The surface is profiled again without the gold layer. To this end, the profiles of gold conductors cured with a laser power of 0.2 to $1 \mathrm{~W}$ and curing velocities of $0.1,1,2.2$, and $3.4 \mathrm{~mm} / \mathrm{s}$ are investigated.

Figure 8 shows the cross-sectional areas of gold lines produced with a curing power of $0.8 \mathrm{~W}$ and curing velocities of 1.0 (Fig. 8a), and $3.4 \mathrm{~mm} / \mathrm{s}$ (Fig. 8b), with (solid line) and without (dotted line) a gold layer. It is not possible to profile exactly the same spot twice because the sample has to be removed from the profiler to remove the gold layer, but the method still gives good results despite this.

The glass substrates showed no deformation for laser power smaller than $0.4 \mathrm{~W}$ for all curing velocities, resulting in "bowl-shaped" cross-sectional areas (Fig. 4a, c). For laser power of $0.4 \mathrm{~W}$, a small bump starts to evolve in the center of the line. The slowest curing velocity of $0.1 \mathrm{~mm} / \mathrm{s}$ leads for laser power larger than $0.4 \mathrm{~W}$ and to destruction of the glass substrate. Cross-sections produced at the same laser power but different curing velocities show a decrease of line width with

increasing velocity, but a growth of the height of the bump in the center region. This phenomenon intensifies with higher power and reaches a maximum at a laser power of $1.2 \mathrm{~W}$, where the height of the bump in the center region is even higher than the lateral rims and reaches a maximum of almost ten times the rim size. The bumps of all lines are completely formed by a volume gain of the glass substrate (without undercutting). A similar phenomenon of bump formation during $\mathrm{CO}_{2}$ laser texturing of silicate glasses was observed and investigated by Bennett $[11,14,15]$ as well by Shiu $[16,17]$, which was found to be a product of volume expansion (without any recession of material below the surface). The proposed mechanism [11] for the volume gain is a density reduction in the glass by rapid quenching after melting of the substrate. This explanation holds true and can be adopted in the present work.

The bump formation is a phenomenon which is related to the properties of glass substrates. The explanation can be found by investigating the behavior of glass during melting/solidification. Glasses are disordered materials without a periodic crystalline structure but exhibit mechanical properties comparable to solids. The most common way of making a glass form a melt is by cooling the viscous liquid material fast enough to avoid crystallization [18] ("supercooling"). Figure 9a shows the phase diagram of glass in which the volume of a liquid is plotted as a function of temperature at a constant pressure. The glassy state is a metastable thermodynamic state which is reached during the cooling down of 

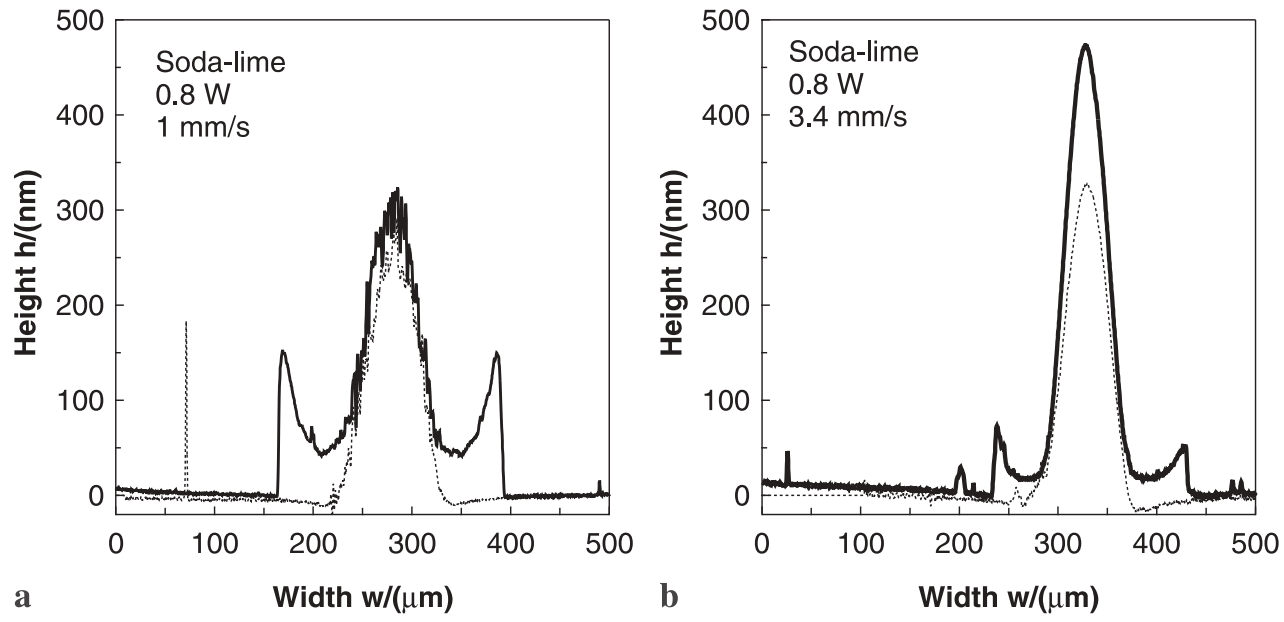

FIGURE 8 Cross-sectional areas with (solid line) and without gold layer (dotted line) of gold conductors cured with a laser beam radius of $39 \mu \mathrm{m}$, a laser power of $0.8 \mathrm{~W}$, and a curing velocity of: (a) $1.0 \mathrm{~mm} / \mathrm{s}$ and (b) $3.4 \mathrm{~mm} / \mathrm{s}$ the liquid glass, a thermodynamically stable phase, below the freezing temperature $T_{\mathrm{m}}$. The thermodynamically stable solid state would be the crystalline phase which can be reached by cooling down the liquid glass very slowly following the equilibrium line (dash-dot line in Fig. 9). When the cooling process is fast, the molecules cannot rearrange themselves and adjust to the current temperature condition, so that crystallization cannot be obtained because the molecular motion is slowing down with cooling below the freezing point $T_{\mathrm{m}}$, resulting in a glass with the frozen structure of the liquid. The slower the cooling rate, the lower the achievable temperature becomes before departing from the liquid state equilibrium. Consequently, the glass transition temperature $T_{\mathrm{g}}$ increases with the cooling rate [18].

Depending on the cooling rates, the cooling path is moving along constant "fictive temperature" lines (dashed lines in Fig. 9b). The fictive temperature of a glass is the temperature at which a given "frozen-in" microstructure would be in a thermodynamic equilibrium. States of the same fictive temperature have the same microstructure which is the microstructure of the glass at the transition temperature. Departure from the equilibrium line, onto a constant fictive temperature line occurs at the transition temperature. The liquid-glass transition is therefore not an equilibrium process and results in a glass with properties dependent on the cooling history. Consequently, the glass transition temperature should be thought of as identifying the temperature at which the rate of change of microstructure in the glass is comparable to the rate of temperature change. The transition temperature moves to lower values when the rate of changes becomes slow, and moves to higher values when the rate of change becomes fast. The transition temperature is also defined as the temperature at which the viscosity, $\eta$, is equal to $10^{13}$ poise.

In the process of laser curing with high power the glass temperature is elevated along a constant fictive line from the glassy state " $a$ " to the liquid " $c$ ". The heating path is departing from this fictive temperature line at the crossing "e", at the transition temperature $\left(T_{\mathrm{g} 1}\right)$, and converges with the equilibrium line and the final state "c" is reached by following the equilibrium line. During the cooling, the path remains on the equilibrium line until the transition temperature is reached again. Since cooling is faster than in the process of manufacturing of the glass substrate the transition temperature is higher $\left(T_{\mathrm{g} 2}\right)$ and the path is following the fictive temperature line to the end state "d" (Fig. 9b). The specific volume of the glass is larger after the curing process compared to the non-heat treated glass. The thermodynamic temperature has to exceed the corresponding transition temperature in order to soften the material and allow for a volumetric change. The increasing bump heights with increasing laser power can be explained by a larger penetration depth of the heat into the substrate (more material is softened and experiences a volume
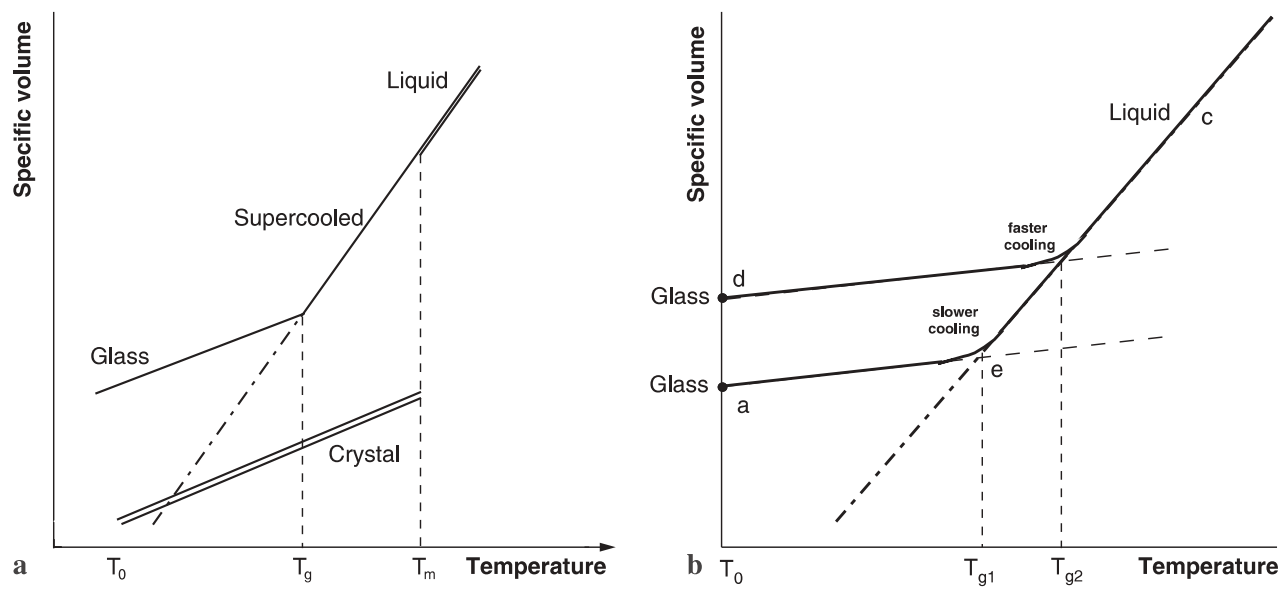

FIGURE 9 (a) Phase diagram of glass $(v-T$-diagram $) . \quad T_{\mathrm{m}}$ freezing temperature, $T_{\mathrm{g}}$ glass transition temperature and $\mathrm{T}_{0}$ ambient temperature. The double lines indicate stable states and the slash-dotted line is the equilibrium line. (b) Phase diagram of glass. Heating and cooling cycle (qualitatively) "a-c-d". Transition temperature $\left(T_{\mathrm{g} 2}\right)$ and the glass volume are higher for a sample that has been cooled rapidly relative to one cooled slowly $\left(T_{\mathrm{g} 1}\right)$. Therefore, glass samples of the same composition but with different cooling histories will have different $298 \mathrm{~K}$ densities 
gain). The reason for increasing bump height with increasing curing velocity is similarly explained. The cooling rate of a curing process with a curing velocity of $1 \mathrm{~mm} / \mathrm{s}$ is smaller than the cooling rate of a curing velocity of $3.4 \mathrm{~mm} / \mathrm{s}$. Therefore, the transition temperature of the process with a curing velocity of $3.4 \mathrm{~mm} / \mathrm{s}$ is higher than the one of the $1 \mathrm{~mm} / \mathrm{s}$ process. Hence, the glass specific volume of the process with a curing velocity of $3.4 \mathrm{~mm} / \mathrm{s}$ is slightly higher than the one with a curing velocity of $1 \mathrm{~mm} / \mathrm{s}$ resulting in a higher bump. In the case of a laser power of $0.2 \mathrm{~W}$ the actual thermodynamic temperature does not reach the transition temperature and no bump is generated.

The validity of the above explanation can be tested experimentally by the employment of a chemically strengthened glass or crystalline quartz generally having a higher softening temperature, and numerically by calculating the temperature inside the substrate. In Table 3 the softening temperature and thermal conductivity of the employed substrates, soda-lime glass (objective slides), AF 45 (modified borosilicate glass used for LCD's, electroluminescence displays, CCD covers, solar cells, hybrid switches), and crystalline quartz (wafer) are given. Because the softening and melting does not occur exactly at a certain point but over a temperature range, it is difficult to define the melting and softening temperatures. However, as the viscosity of glass varies continuously from the molten state to the glassy state commonly accepted viscosity reference points are defined. The softening temperature is defined as the temperature where the viscosity is equal to $10^{7.6} \mathrm{~Pa}$ s. Comparing the softening temperature of soda-lime glass with AF 45 and quartz yields a difference of 200 and $1000^{\circ} \mathrm{C}$ respectively. If the assumptions of the fictive temperature map are correct, the bump formation for the AF 45 cured with a laser power of $0.8 \mathrm{~W}$ should be smaller than that of soda-lime glass. In Fig. 10 the cross-sectional profiles with and without gold layers and the calculated isotherms of the two substrates a) soda-lime glass and b) AF 45 are compared for a laser power of $0.8 \mathrm{~W}$ and a curing velocity of $1.0 \mathrm{~mm} / \mathrm{s}$. The bump height of the AF 45 substrate is only half of the soda-lime substrate (Fig. 10b). No bump is generated on the quartz substrate (not shown here), since thermal diffusivity is very high. The bump height is increasing with increasing curing velocities for the AF 45 substrate as for the soda-lime substrate. Because the quartz is not heated up to its transi- tion temperature, no bump forms. The model of Moody and Hendel [19] is adapted for the calculations of the temperature profiles induced by a moving elliptical continuous laser beam with a Gaussian intensity distribution. The employed argon laser beam has a Gaussian intensity distribution. The polarization is perpendicular and the laser spot is elliptical due to the $30^{\circ}$ angle of incidence. The temperature dependent conductivity, diffusivity and specific heat are taken from manufacturer's data. The surface reflectivity of gold is taken because the laser beam is reflected by the gold layer formed ahead of the laser spot, as described earlier in this section. The reflectivity of a metal with an incident laser beam with an incident angle $(\Theta>0)$ and perpendicular polarization is defined as:

$$
\varrho_{\perp}(\Theta)=\frac{(n-\cos \Theta)^{2}+k^{2}}{(n+\cos \Theta)^{2}+k^{2}}
$$

For gold the values $n(\lambda=516 \mathrm{~nm})=0.608$ and $k(\lambda=$ $516 \mathrm{~nm})=2.12$ are taken from Palik [20] giving a total reflectivity of $68 \%$. The reflectivity is an important factor for the heat input. A reduced reflectivity of $50 \%$ leads to an overall temperature increase and the maximal temperature in the center is increased from 1400 to $1700^{\circ} \mathrm{C}$. The calculated isotherms for the two substrates in Fig. 10 are shown as a function of penetration depth. The softening temperatures of soda-lime glass $\left(T_{\mathrm{S}} \approx 700\right)$ and $\mathrm{AF} 45\left(T_{\mathrm{S}} \approx 900\right)$ are indicated by the thick solid line. The width at the surface of the soda-lime substrate where the temperature exceeds the softening temperature is $100 \mu \mathrm{m}$ which is identical to the bump width of the corresponding gold conductor (Fig. 10a). The maximal temperature is $1400^{\circ} \mathrm{C}$ in the center and the penetration depth of the softening isotherm is $35 \mu \mathrm{m}$. The isotherms inside the AF 45 substrate are similar to isotherms of the soda-lime substrate because the thermal properties of the two glasses are almost identical. The softening temperature of the AF 45 is higher and therefore less material experiences a volume gain resulting in a smaller bump. Again the width of the bump is nearly identical with the width of the area where the temperature exceeds the softening temperature (Fig. 10b). With reduced laser power the heat-affected zone is shallower and the extent of the volume subjected to temperature exceeding the softening point is smaller, with

\begin{tabular}{lccc}
\hline Substrate & $\begin{array}{c}\text { Softening temperature }\left(T_{\mathrm{S}}^{*}\right) / \\
\text { Temperature limit }\left[{ }^{\circ} \mathrm{C}\right]\end{array}$ & $\begin{array}{c}\text { Thermal conductivity } \\
{[\mathrm{W} /(\mathrm{m} \mathrm{K})]}\end{array}$ \\
\hline Soda-lime glass & $696^{\mathrm{a})}$ & $0.9^{\mathrm{c})}$ & $\left(24^{\circ} \mathrm{C}\right)$ \\
AF 45 & $883^{\mathrm{b})}$ & $0.9^{\mathrm{c})}$ & $\left(24^{\circ} \mathrm{C}\right)$ \\
Quartz (crystalline) & $1683^{\mathrm{c})}$ & $12^{\mathrm{c})}$ & $\left(0^{\circ} \mathrm{C}\right)$ \\
Si & & $148^{\mathrm{c})}$ & $\left(27^{\circ} \mathrm{C}\right)$ \\
Resin printing plate $(1 \mathrm{~mm})$ & 105 & \multicolumn{2}{c}{0.19} \\
Epoxy printing plate $(1.5 \mathrm{~mm})$ & 130 & \multicolumn{2}{c}{0.45} \\
Polyimide $(25 \mu \mathrm{m})$ & 260 & & \\
\hline
\end{tabular}

a) Schott specification data sheet PCE - TKT for B 270 Superwite (glass with material constants comparable to the soda-lime objective slides)

b) Schott specification data sheet for PCE - DGS for AF 45

c) CRC Handbook of Chemistry and Physics

TABLE 3 Softening temperature $T_{\mathrm{S}}$ and thermal conductivity of the typical glass temperatures of soda-lime glass (objective slides), AF 45 , and crystalline quartz (wafer). The softening temperatures correspond to an accepted viscosity reference point $\left({ }^{*} \eta=10^{7.6} \mathrm{~Pa}\right.$ s). Temperature limit and thermal conductivity of substrates commonly used in electronics: resin printing plate, epoxy printing plate, and polyimide 

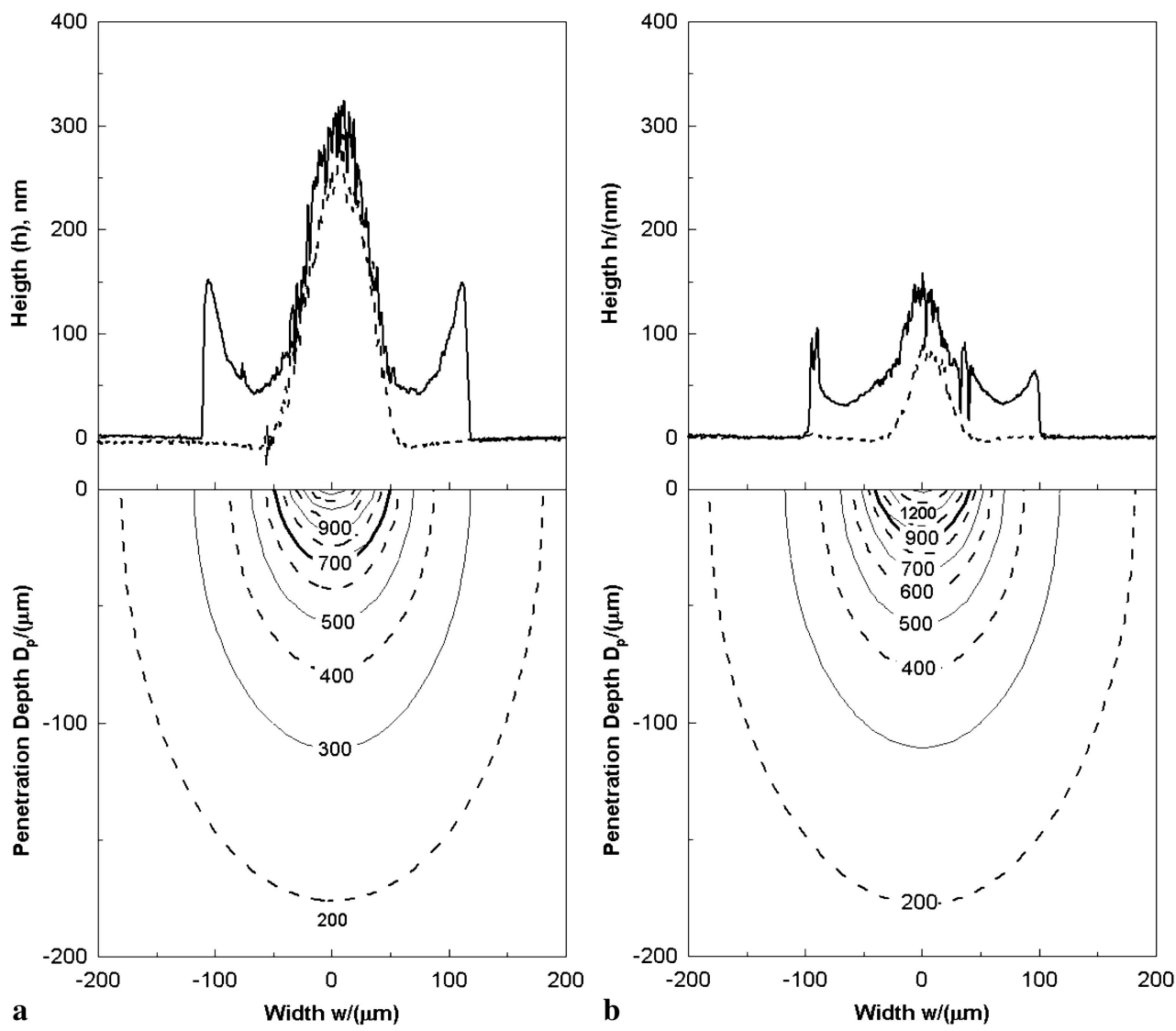

FIGURE 10 Top: Cross-sectional profiles of gold line cured with a laser beam radius of $39 \mu \mathrm{m}$, a laser power of $0.8 \mathrm{~W}$ and a curing velocity of $1 \mathrm{~mm} / \mathrm{s}$ on (a) soda-lime glass and (b) AF 45 (chemically strengthen glass). Bottom: Temperature isotherms as a function of penetration depth in the substrate calculated for a laser with identical parameter as in the experiment. The softening temperature (thick solid line) is (a) $700{ }^{\circ} \mathrm{C}$ (soda-lime glass) and $900^{\circ} \mathrm{C}(\mathrm{AF} 45)$. The maximal temperature is $1400{ }^{\circ} \mathrm{C}$ correspondingly reduced bump height. If the softening temperature is not reached no bump is generated. In both cases the calculated temperature profiles in the substrate confirm the theory that the bump formation is a product of volume expansion due to a density reduction in the glass by rapid quenching after melting, and the bump height is proportional to the penetration depth of the "softening" temperature. The deviations between experiments and calculations can be explained by the approximate nature of the model, (the model describes a laser only heating a substrate without a gold layer on top) and the uncertainties of the material properties, i.e. reflectivity, conductivity and diffusivity. The reflectivity plays an important role, as explained before, on how much energy is absorbed by the substrate. Topography (texturing) is usually generated in metal surfaces melted by lasers, which affects the optical behavior and hence the energy coupling to the target material. Zhang et al. [21] proposed a reflectivity reduction of $26 \%$ for a gold layer close to the melting point. Nevertheless, the model shows reasonable results and is therefore a good tool to evaluate production parameters, i.e. laser power and curing velocity, for a given laser diameter and for different materials after appropriate adjustments of material constants.

In Fig. 11 the resistivities of gold conductors cured on soda-lime glass substrates for laser power of 0.4 (Fig. 11a) and $0.8 \mathrm{~W}$ (Fig. 11b) as a function of curing velocity, are compared with the resistivities of gold conductors cured on AF 45 and quartz (crystalline) substrates. The AF 45 shows for both laser powers a better resistivity than the soda-lime glass because the bumps in both cases are smaller. The quartz substrate is expected to have an even better resistivity than the AF 45 substrate but for a laser power of $0.4 \mathrm{~W}$ only one single resistivity can be measured at the low curing velocity of $0.1 \mathrm{~mm} / \mathrm{s}$, indicating that the heat input at $0.4 \mathrm{~W}$ is not sufficient to produce gold conductors on quartz. The thermal conductivity (Table 3 ) of the quartz substrate is an order of magnitude higher than that of the glass substrates (soda-lime and AF 45), therefore the heat diffuses faster into the quartz substrate. Even for a laser power of $0.8 \mathrm{~W}$, the resistivities for the quartz substrate are of the same order of magnitude as the AF 45 (Fig. 11b). Experiments with quartz substrate for the highest possible laser power $(1.2 \mathrm{~W})$ (Fig. $11 \mathrm{~b})$, give drastically reduced resistivities, also for high curing velocities, but still the energy input is not sufficient to achieve the same conductor quality as was possible with AF 45. An experiment using $\mathrm{Si}$ wafer as the substrate was not successful for the same reason of very high thermal conductivity (Table 3 ). Even the maximal laser power available of $1.2 \mathrm{~W}$ is not enough to create gold conductors.

Resistivity measurements for soda-lime glass for laser power between 0.2 (minimal laser power) and $1.0 \mathrm{~W}$ as a function of curing velocity ( 0.1 to $3.4 \mathrm{~mm} / \mathrm{s}$ ) showed the same trend of increasing resistivity with increasing curing velocity. However, the curing velocities have different meanings for the different powers. For a laser power of $0.2 \mathrm{~W}$ the maximal curing velocity is reached with $2.4 \mathrm{~mm} / \mathrm{s}$ meaning that at this velocity the time for heat input is too short to allow a complete sintering of the gold nanoparticles, hence, the resistivity is increasing drastically. Generally, the resistivity of 


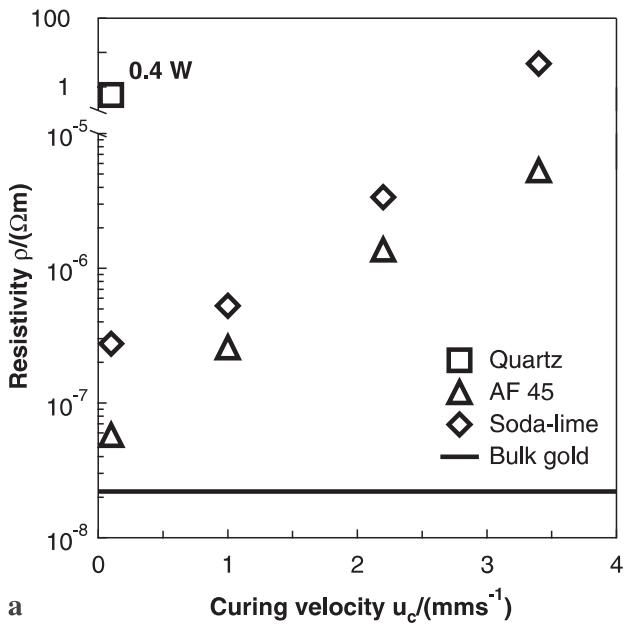

the gold lines cured with $0.2 \mathrm{~W}$ is higher than that of the lines cured with higher power due to less complete sintering of the nanoparticles due to the smaller energy input. For soda-lime glass a laser power of $0.4 \mathrm{~W}$ yields the lowest overall resistivity for all curing velocities. This can be explained by the fact that the bumps are small, because the penetration depth where the transition temperature is reached is smaller than with higher laser power. For this power, the reason for the decrease in resistivity with increasing curing velocity must be a combination of growing bump height and incomplete sintering of the gold nanoparticles due to insufficient heat input. For soda-lime substrates a laser power of $0.4 \mathrm{~W}$ and a curing velocity of 1 to $2 \mathrm{~mm} / \mathrm{s}$ are determined as the best parameter combination.

The employment of nanoparticles with their reduced melting temperature and their localized melting with an appropriately selected laser should, if necessary, minimize or even prevent thermal damage to the substrate. Therefore, the feasibility of producing gold conductors on more delicate substrates is examined. Three different synthetic substrates commonly used as base plates for IC's or flexible connections between electronic parts, resin (rigid), epoxy (rigid), and polyimide (flexible), are listed with the maximal supported temperature and thermal conductivity in Table 3.

From the experiments with glass substrates, the gold layer etching experiments are especially helping to estimate the temperature load to the substrate. A qualified laser power range of $0.1 \mathrm{~W}$ (with slow curing velocities) to a maximal $0.3 \mathrm{~W}$ (with high curing velocities) is chosen in order to prevent a burning of the substrate. The thermal conductivity of the synthetic substrates is two to five times smaller than in the case of glass. Hence, the energy losses to the substrate are smaller and a larger portion of the input energy is utilized for the sintering process. It is possible to produce conducting gold lines on all substrates, but it is not possible to reliably measure any profiles because even with low power all the substrates are deforming. This results in valley profiles giving no reliable information about the actual cross section of the gold line, and therefore the resistivity cannot be calculated. Nevertheless it was shown that the process is potentially applicable to polymeric substrates employing laser heating, indicating that a temporal modulation of the beam could be an effective strategy to improve the technique.
FIGURE 11 Resistivities of gold lines cured with a laser beam radius of $39 \mu \mathrm{m}$, a laser power of (a) $0.4 \mathrm{~W}$ and (b) $0.8 \mathrm{~W}$ as a function of curing velocity for different substrates: quartz (square), AF 45 (triangle), and soda-lime (diamonds). The solid line represents the resistivity of bulk gold. In diagram (b) the resistivity of gold lines cured with a laser power of $1.2 \mathrm{~W}$ is shown additionally
4

\section{Summary}

This paper presented an experimental investigation of the process of deposition and moderate-temperature laser annealing of nanoparticle inks, related to a novel technique of producing electrically conductive gold stripes of thicknesses of 10-100 nm and widths of 10-500 $\mu \mathrm{m}$. A dropon-demand ink jet is employed to print the solvent loaded with gold nanoparticles in micro-lines onto a flat substrate. Irradiated laser thermal energy is used to evaporate the solvent and to initiate the melting and curing process of the nanoparticles. The subsequent heating and melting of the nanoparticles is achieved by heat diffusion from the gold layer formed previously through the substrate to the leading edge of the material. The surrounding liquid is heated and finally evaporated through thermal diffusion from the heated particles. The microstructures produced are analyzed with scanning force microscopy (SFM) and energy-dispersive $\mathrm{X}$-ray analysis (EDX). Etching experiments, where the crosssectional area of the gold conductor is profiled with and without a gold layer, revealed an irreversible thermal expansion of the glass substrate causing a bump formation in the center of the gold conductor at high laser powers. The phase diagram of glass is utilized to explain the volume gain of the glass. The bump grows with increasing velocity due to higher quenching velocity. Calculations of the substrate temperature profiles induced by a scanning continuous Gaussian laser beam, confirm the transgression of the glass softening temperature within the bumpy region which is the precondition for a glass expansion. The bump formation is shown to be reduced by the employment of chemically strengthened glass with higher softening temperature.

Additional experiments with $\mathrm{Si}$ and $\mathrm{SiO}_{2}$ (crystalline quartz) substrates demonstrated the importance of thermal diffusion in the curing process. The thermal conductivities of both materials are larger than the thermal conductivity of glass. Therefore, the heat induced by the laser diffuses faster into the substrate, effectively providing less heat for the melting/curing process. In this context, a laser power which would permanently deform the glass substrate is not sufficient to heat up the $\mathrm{Si}$ or $\mathrm{SiO}_{2}$ substrate to produce sustainable gold conductors. 
The resistivities of the produced gold conductor stripes are in general higher than those of bulk gold. The fact that the gold layers produced in this novel process are of the order of a hundred nanometers, means that this result is satisfactory. Future research will aim at a further reduction of the resistivity.

ACKNOWLEDGEMENTS This work was supported in part by the Swiss National Science Foundation (Grant No. 2000-063580.00) and by the US National Science Foundation under Grant CTS-0417563. The support of M. Dietzel with respect to the computational work on the temperature profiles is kindly acknowledged.

\section{REFERENCES}

1 P. Buffat, J.P. Borel: Phys. Rev. A 13, 6 (1976)

2 N.R. Bieri, J. Chung, S.E. Haferl, D. Poulikakos, C.P. Grigoropoulos: Appl. Phys. Lett. 82, 20 (2003)

3 S. Haferl, D. Poulikakos: J. Appl. Phys. 92, 3 (2002)

4 S. Haferl, Z. Zhao, J. Giannakouros, D. Attinger, D. Poulikakos: Rev. Heat Transf. XI, 65 (2000)

5 D. Attinger, Z. Zhao, D. Poulikakos: J. Heat Transf. - Trans. ASME 122, $544(2000)$
6 D.B. Bogy, F.E. Talke: IBM J. Res. Dev. 28, 3 (1984)

7 J.F. Dijksman: J. Fluid Mech. 139, 173 (1984)

8 S. Haferl, D. Poulikakos: Int. J. Heat Mass Transf. 46, 3 (2003)

9 A.L. Yarin, D.A. Weiss: J. Fluid Mech. 283, 141 (1995)

10 J. Chung, S.H. Ko, N.R. Bieri, C.P. Grigoropoulos, D. Poulikakos: Appl. Phys. Lett. 84, 5 (2004)

11 T.D. Bennett, D.J. Krajnovich, L. Li, D.J. Wan: J. Appl. Phys. 84, 5 (1998)

12 N.R. Bieri, J. Chung, D. Poulikakos, C.P. Grigoropoulos: Superlattices Microstruct. 35, 437 (2004)

13 J. Chung, N.R. Bieri, S. Ko, C.P. Grigoropoulos, D. Poulikakos: Appl. Phys. A 79, 1259 (2004)

14 T.D. Bennett, D.J. Krajnovich, L. Li: J. Appl. Phys. 85, 1 (1999)

15 T.D. Bennett, L. Li: J. Appl. Phys. 89, 2 (2001)

16 T.R. Shiu, C.P. Grigoropoulos, D.G. Cahill, R. Greif: J. Appl. Phys. 86, 3 (1999)

17 T.R. Shiu, C.P. Grigoropoulos, R. Greif: J. Heat Transf. - Trans. ASME 121, 4 (1999)

18 P.G. Debenedetti, F.H. Stillinger: Nature 410, 6825 (2001)

19 J.E. Moody, R.H. Hendel: J. Appl. Phys. 53, 6 (1982)

20 E.D. Palik: Handbook of Optical Constants of Solids II (Academic Press, San Diego 1991)

21 X. Zhang, S.S. Chu, J.R. Ho, C.P. Grigoropoulos: Appl. Phys. A 64, 545 (1997) 